\title{
Analysis of a dynamic model of guard cell signaling reveals the stability of signal propagation
}

\author{
Xiao Gan and Réka Albert
}

\begin{abstract}
Background: Analyzing the long-term behaviors (attractors) of dynamic models of biological systems can provide valuable insight into biological phenotypes and their stability. In this paper we identify the allowed long-term behaviors of a multi-level, 70-node dynamic model of the stomatal opening process in plants.

Results: We start by reducing the model's huge state space. We first reduce unregulated nodes and simple mediator nodes, then simplify the regulatory functions of selected nodes while keeping the model consistent with experimental observations. We perform attractor analysis on the resulting 32-node reduced model by two methods: 1. converting it into a Boolean model, then applying two attractor-finding algorithms; 2. theoretical analysis of the regulatory functions. We further demonstrate the robustness of signal propagation by showing that a large percentage of single-node knockouts does not affect the stomatal opening level.

Conclusions: Combining both methods with analysis of perturbation scenarios, we conclude that all nodes except two in the reduced model have a single attractor; and only two nodes can admit oscillations. The multistability or oscillations of these four nodes do not affect the stomatal opening level in any situation. This conclusion applies to the original model as well in all the biologically meaningful cases. In addition, the stomatal opening level is resilient against singlenode knockouts. Thus, we conclude that the complex structure of this signal transduction network provides multiple information propagation pathways while not allowing extensive multistability or oscillations, resulting in robust signal propagation. Our innovative combination of methods offers a promising way to analyze multi-level models.
\end{abstract}

Keywords: Network model, Discrete dynamic model, Biological network, Signal transduction, Plant signaling, Attractor, Stomatal opening, Network reduction, Boolean conversion, Stable motif

\section{Background}

Modeling offers a comprehensive way to understand biological processes by integrating the components involved in them and the interactions between components. Models can recapitulate and explain the emergent outcome(s) of the process $[1,2]$. Representing cellular processes that involve many proteins and small molecules by a signal transduction network can reveal indirect relationships between components and provide new insight [3-5]. Such network usually consists of nodes representing biological entities, and edges representing interactions. Once a network has been constructed, dynamic

\footnotetext{
*Correspondence: rza1@psu.edu

Department of Physics, The Pennsylvania State University, University Park, PA, USA
}

modeling, where each node in the network is associated with a variable representing its abundance or activity, can further describe the behavior of the network. Dynamic models can have continuous variables whose change is described by differential equations [6], discrete variables described by discrete (logical) regulatory functions $[7,8]$, or a combination of continuous and discrete variables [9]. The major advantage of discrete dynamic and continuous-discrete hybrid models is that they use many fewer parameters than continuous models and thus need less parameter estimation [10-12]. Modeling allows one to analyze the biological system represented by the network in silico, when performing the relevant experiment is infeasible. It also helps identify general principles of biological systems [13, 14]. 
The biological process of stomatal opening in plants is a good example of a complex system wherein modeling leads to significant gain in understanding $[15,16]$. Stomata are pores on leaf surfaces that allow the plant to exchange carbon dioxide $\left(\mathrm{CO}_{2}\right)$ and oxygen with the atmosphere. Stomata are formed by two guard cells that can change shape: swelling of guard cells leads to stomatal opening; their shrinking leads to stomatal closure. The shape of each guard cell is directly controlled by water flow through the membrane, which is in turn controlled by ion flow. Different signals can affect the guard cell, changing its ion concentration in direct and indirect ways, resulting in stomatal opening or closure [17-19]. These signals include light of different wavelengths, $\mathrm{CO}_{2}$ concentration in the air, and plant hormones like abscisic acid (ABA). The regulation of stomatal opening is essential to plants, as it controls vital activities like the uptake of $\mathrm{CO}_{2}$ for photosynthesis, and the unavoidable water loss through evaporation [20]. Through extensive experimentation over several decades, more than 70 proteins and small molecules have been identified to participate in this process.

Sun et al. [15] recently constructed a signal transduction network based on conclusions from more than 85 articles in the literature, describing how more than 70 nodes (proteins, small molecules, ions) interact with each other in the stomatal opening process. The network, reproduced as Fig. 1 [15], includes four source nodes that correspond to the signals red light, blue light, $\mathrm{CO}_{2}$, and ABA. The more than 150 edges are directed and signed, with arrowheads indicating activation and terminal black circles indicating inhibition.

Translating this network into a dynamic model, Sun et al. characterized each node with a discrete variable describing its activity and with a discrete (logical) regulatory function describing its regulation. Twenty-one out of the 70 nodes in the model are multi-level, the rest are Boolean (binary). The levels reflect relative and qualitative information: a level of 2 is a higher level than 1 , but should not be interpreted as twice as high. A few

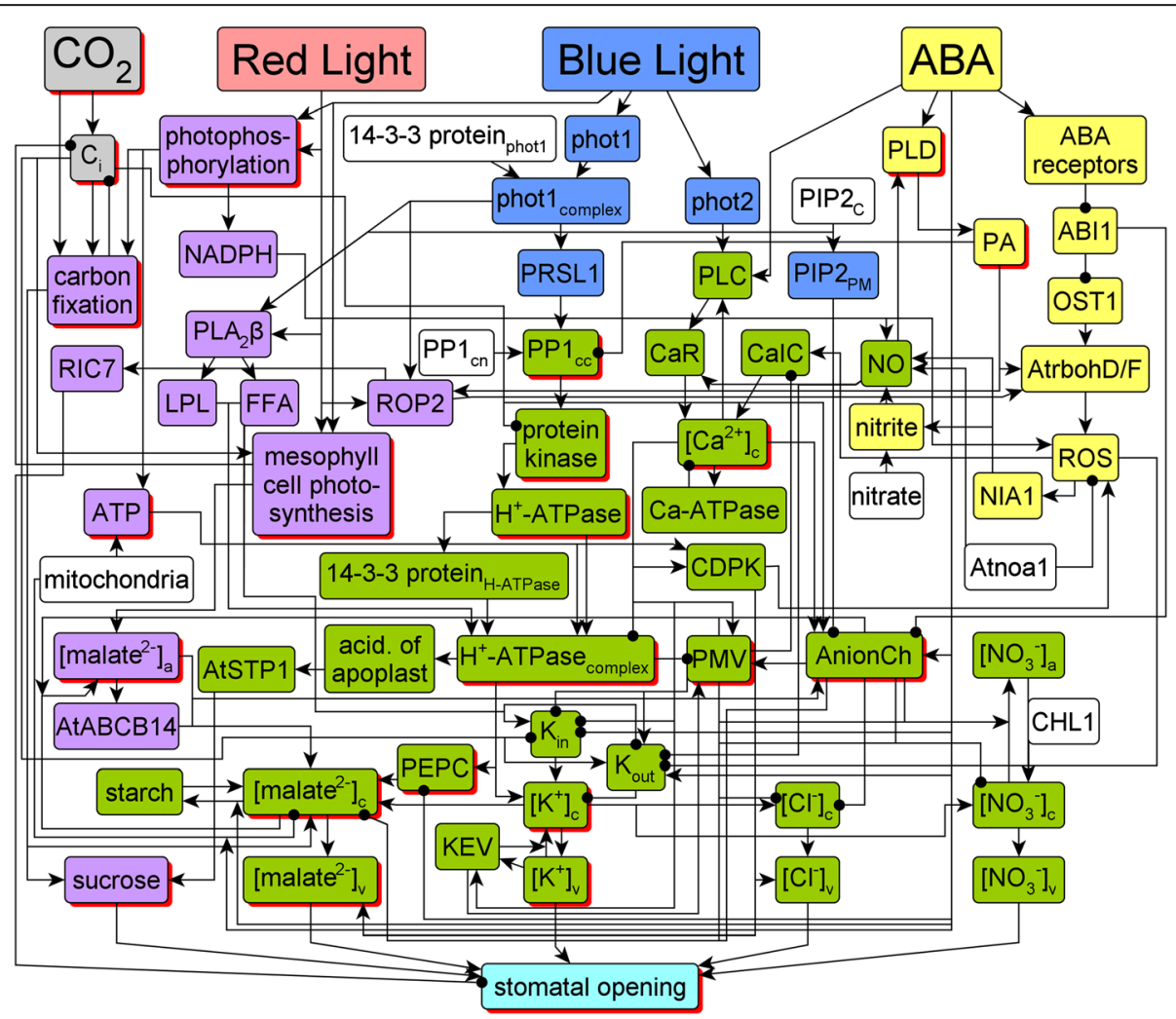

Fig. 1 The signal transduction network responsible for stomatal opening, as reconstructed by Sun et al. [15]. The color of a node marks which signal regulates this node. Red nodes are regulated solely by red light. Blue nodes are regulated solely by blue light. Yellow nodes are regulated solely by $\mathrm{ABA}$. Grey nodes are regulated by $\mathrm{CO}_{2}$. Purple nodes are regulated by both blue and red light. Green nodes are regulated by blue (and potentially, red) light and ABA. White nodes are source nodes not regulated by any of the four signals. To improve visualization, certain pairs of edges with the same starting or end nodes overlap. Nodes with multiple levels in the dynamic model are represented by red shadows; the others are Boolean. The full names of the network components denoted by abbreviated node names are given in Table 1. This figure and part of its caption is reproduced from Sun Z, Jin X, Albert R, Assmann SM (2014) Multi-level Modeling of Light-Induced Stomatal Opening Offers New Insights into Its Regulation by Drought. PLoS Comput Biol 10(11): e1003930. doi:10.1371/journal.pcbi.1003930 
discrete values are not integers; e.g. stomatal opening is a weighted sum with non-integer weights. The dynamic model has $\sim 10^{31}$ states. The logical regulatory functions, describing each node's future state based on the states of the node's regulators, use a combination of Boolean logic operators (And, Or, Not), algebraic operations, and input-output tables. For example, the regulatory function of PRSL1 is:

\section{PRSL1 $^{*}=\operatorname{phot}_{\text {complex }}$ Or phot2.}

Here for simplicity the node states are denoted by the node names; the asterisk in "PRSL1" indicates that this will be the next state of the PRSL1. The "Or" Boolean operator expresses that either of the blue light receptors, i.e. the phot 1 complex or phot 2 , can independently activate PRSL1.

The Sun et al. model starts from an initial condition representative of closed stomata. Then a combination of the four input signals is applied. Red light, blue light, and $\mathrm{ABA}$ are represented as binary variables, and external $\mathrm{CO}_{2}$ is represented with three states: $0\left(\mathrm{CO}_{2}\right.$ free air), 1 (ambient $\mathrm{CO}_{2}$ ) and 2 (high $\mathrm{CO}_{2}$ ). The system's response is simulated through repetitive re-evaluation of each node's state until a stable value of stomatal opening is observed. The model successfully captures stomatal opening in response to combinations of the signals. It also successfully reproduces stomatal opening under most of the experimentally studied perturbation scenarios (i.e. genetic knockouts or external supply of components). In total, the model is consistent with 63 out of 66 experimental observations collected by Sun et al. [15]. The model predicts the outcome of a large number of scenarios that have not been explored experimentally so far. It also revealed a gap of knowledge regarding the cross-talk of red light and ABA signaling, and filled it with a newly predicted interaction.

Although the Sun et al. model recapitulates existing knowledge and offers new predictions, the model's full dynamic repertoire could not be characterized due to its large state space. Instead, Sun et al. focused on tracking the output node, stomatal opening, and a few selected internal nodes, in time. In this paper we apply multiple methods to analyze the model and aim to fully map all its potential long-term behaviors, or in other words, attractors.

\section{Methods}

\section{Attractors of a dynamical system}

An attractor is a set of states from which only states in the same set can be reached. Attractors that consist of a single state are called stable steady states or fixed points; attractors that contain multiple states are called complex attractors or oscillations [10]. In biological networks, attractors often have significant biological meaning. In a cell signaling network, attractors correspond to cell types, cell fates or behaviors [21]. For example, one attractor can represent a healthy differentiated cell, while another attractor can represent an abnormally motile cancer cell [22].

\section{Update scheme of a discrete time model}

In the Sun et al. model, as in most discrete dynamic models, time is an implicit variable. As there is very little information about the kinetics of the nodes in the stomatal opening network, the model incorporates an element of stochasticity in timing. The timing does not affect a system's fixed point attractors, but it can change the complex attractors and the possibility of reaching a given attractor from a given initial state [10]. In the Sun et al. model, a random-order asynchronous update is used. Specifically, at each time step, a random order of nodes (excluding the four input nodes and the output node stomatal opening) is generated, and each node's state is reevaluated in this order; stomatal opening is always updated last. In the next time step a different order is generated randomly. In this paper, we use a different type of stochastic update, called general asynchronous update, wherein a randomly selected node is updated at each time step. This is required by the network reduction method we use. Although this theoretically could cause a difference in complex attractors, we will show that in this specific model the two update methods yield the same attractors.

\section{Network reduction}

To reduce the Sun et al. model's state space, we apply a network reduction method developed by Saadatpour et al. [23] that is proven to preserve the attractors of a Boolean model. Two types of nodes can be reduced (eliminated or merged): source nodes with no incoming edges, and simple mediator nodes that have one incoming and/or one outgoing edge. In the reduction, the source node's state is directly plugged into the regulatory function of all of its direct successor nodes; then the source node is eliminated. For a simple mediator node with one predecessor (regulator) and one successor (target), its regulator is connected to its target and the mediator node is merged into the regulator. If there is one regulator and several targets of the mediator node, but no direct edges between the regulator and any of the targets, the mediator node is merged into the regulator. Conversely, if there are several regulators and one target of the mediator node, but no direct edges among any of the regulators and the target, the mediator node is merged into its target. Although this method is not proven in the multi-level case, we conjecture that attractors are also conserved for a multi-level model, and will 
show from the results that in the Sun et al. model this reduction method preserved all attractors.

\section{Elimination of redundant edges}

During the process of creating a discrete dynamic model from biological data, when an influence is weaker than other influences, the modeler may choose to omit this influence or, alternatively, include it a redundant way. The latter choice was made by Sun et al. in four cases, leading to four regulatory functions that contain an input that does not affect the outcome of the regulatory function. One of these is

\section{ROS $^{*}=$ NADPH And AtrbohD/F Or NADPH And AtrbohD/F And CDPK Or Not Atnoal}

The italicized words "And", "Or" and "Not" are Boolean logic operators; the non-italicized words represent node names. In this regulatory function every node is Boolean (binary). The first clause "NADPH And AtrbohD/F" and the second "NADPH And AtrbohD/F And CDPK" are connected with an "Or" rule, with the result that the node "CDPK" does not have any influence on the outcome. Therefore, we can prune the edge from CDPK to ROS without changing the model's dynamics. We similarly prune three additional redundant edges.

\section{Converting a multi-level model to Boolean}

There are several possibilities to convert a multi-level model to Boolean [24]. The standard method used in the case of logical models of regulatory networks is the Van Ham mapping $[25,26]$. It preserves the dynamics of the original model if the variables in the original model can be represented by integers and if the original model only allows state transitions in which one node changes its state by one level [26]. The Sun et al. model does not satisfy these criteria. However there still is a conclusion that we can use: All types of conversions maintain the fixed points and the reachability of states (i.e. if there is a sequence of state transitions from state A to state B before conversion, there must be a sequence of state transitions from the corresponding state A' to state B' after the conversion) [26]. So the worst distortion of attractors due to the conversion is the merging of two complex attractors into one. In this light we choose to use an economic mapping of each multi-level node into as many Boolean nodes as necessary for the binary representation of the corresponding integer. We will show that in this specific model, the conversion did not change the attractors.

\section{Abbreviations}

Table 1 summarizes the full names of the network components denoted by abbreviated node names in Fig. 1 .
The same abbreviations are used in the original Sun et al. model and the reduced model developed in this paper.

\section{Results}

\section{Network reduction}

The Sun et al. model has a huge state space of $\sim 10^{31}$ states, making its analysis difficult. To obtain a smaller state space, we reduce the size of the network by applying a network reduction technique developed by Saadatpour et al. [23] that is proven to preserve the attractors of Boolean models (see Methods). All source nodes other than the four signals (blue light, red light, $\mathrm{CO}_{2}$, and abscisic acid) and all simple mediator nodes are identified and reduced. This process is done iteratively until it cannot be done any more. A total of 7 source nodes (14-3-3 proteinphot1, PIP $2_{\mathrm{C}}$, AtNOA1, Nitrate, PP1 $1_{\mathrm{cn}}$, mitochondria, and CHL1), and 19 simple mediator nodes (phot1, phot2, NIA1, $\mathrm{H}^{+}$-ATPase, LPL, ATP, acid. of apoplast, $\left[\mathrm{NO}_{3}^{-}\right]_{\mathrm{v}}$, $\left[\mathrm{Cl}^{-}\right]_{\mathrm{v}}, \mathrm{NADPH},\left[\text { malate }^{2-}\right]_{\mathrm{v}}$, PA, ABA receptors, OST1, PRSL1, PIP2 $2_{\mathrm{PM}}$, AtrbohD/F, Nitrite, and phot $1_{\text {complex }}$ ) are eliminated. Several of the simple mediator nodes form linear paths (e.g. phot1, OST1) thus their iterative reduction shortens the linear paths in the network. In addition, 16 of the 19 reduced mediators have a regulatory function of the form " $\mathrm{B}$ " = A". It is intuitive that reduction of this node type preserves the attractors.

We do not eliminate the four signal nodes because we want to simultaneously explore all the combinations of input signals. We also choose to not reduce the five nodes $\left(\mathrm{K}_{\mathrm{in}}, \mathrm{K}_{\text {out }}, \mathrm{K}_{\mathrm{c}}, \mathrm{Ca}^{2+}\right.$-ATPase, mesophyll cell photosynthesis) whose merging with their sole regulator would result in a self-loop (self-regulation), because such selfloops may be difficult to interpret. Two additional nodes with significant biological meaning to the network (sucrose, stomatal opening), are not reduced either.

Another form of network reduction is the elimination of redundant edges (see Methods). After removal of redundant edges, the node CDPK becomes a sink node, thus it can also be eliminated. The reduction of the above-described nodes and redundant edges simplifies the network from 70 nodes to 42 nodes, with an estimated state space of $\sim 10^{22}$ states.

\section{Simplification of regulatory functions}

In order to further reduce the state space from $\sim 10^{22}$ to a manageable size, we grouped state values so that nodes are represented with fewer states. This grouping was guided by the 66 experimental observations summarized in Sun et al.; we aimed to maintain the reduced model's results consistent with these experimental observations.

For example, in the Sun et al. model [15] the regulatory function of Stomatal Opening is a weighted sum of different ions and sucrose: 
Table 1 Full names of the network components denoted by abbreviated node names in Fig. 1

\begin{tabular}{|c|c|c|c|}
\hline Abbreviation & Full name & Abbreviation & Full name \\
\hline $\begin{array}{l}\text { 14-3-3 } \\
\text { protein } \\
\text { ATPase }\end{array}$ & 14-3-3 protein that binds to the $\mathrm{H}^{+}$-ATPase & $\begin{array}{l}14-3-3 \\
\text { protein }_{\text {phot1 }}\end{array}$ & 14-3-3 protein that binds to phototropin 1 \\
\hline ABA & abscisic acid & $\mathrm{AB} \mid 1$ & 2C-type protein phosphatase \\
\hline $\begin{array}{l}\text { acid. of } \\
\text { apoplast }\end{array}$ & the acidification of the apoplast & AnionCh & $\begin{array}{l}\text { anion efflux channels at the plasma } \\
\text { membrane }\end{array}$ \\
\hline AtABCB14 & $A B C$ transporter gene AtABCB14 & Atnoa1 & protein nitric oxide-associated 1 \\
\hline AtrbohD/F & NADPH oxidase D/F & AtSTP1 & $\mathrm{H}$-monosaccharide symporter gene AtSTP1 \\
\hline $\mathrm{Ca}^{2+}$-ATPase & $\begin{array}{l}\mathrm{Ca}^{2+} \text {-ATPases and } \mathrm{Ca}^{2+} / \mathrm{H}+\text { antiporters responsible for } \mathrm{Ca}^{2+} \text { efflux from } \\
\text { the cytosol }\end{array}$ & CalC & inward $\mathrm{Ca}^{2+}$ permeable channels \\
\hline CaR & $\mathrm{Ca}^{2+}$ release from intracellular stores & carbon fixation & $\begin{array}{l}\text { light-independent reactions of } \\
\text { photosynthesis }\end{array}$ \\
\hline CDPK & $\mathrm{Ca}^{2+}$-dependent protein kinases & CHL1 & $\begin{array}{l}\text { dual-affinity nitrate transporter gene } \\
\text { AtNRT1.1 }\end{array}$ \\
\hline$C_{i}$ & intercellular $\mathrm{CO}_{2}$ concentration & FFA & free fatty acids \\
\hline $\mathrm{H}^{+}$-ATPase & $\begin{array}{l}\text { the phosphorylated } \mathrm{H}^{+} \text {-ATPase at the plasma membrane prior to the } \\
\text { binding of the } \mathrm{H}^{+} \text {-ATPase } 14-3-3 \text { protein }\end{array}$ & $\begin{array}{l}\mathrm{H} \\
{ }^{+} \text {-ATPase } \\
\text { complex }\end{array}$ & 14-3-3 protein bound $\mathrm{H}^{+}$-ATPase \\
\hline KEV & $\mathrm{K}^{+}$efflux from the vacuole to the cytosol & $\mathrm{K}_{\text {in }}$ & $\begin{array}{l}\mathrm{K}^{+} \text {inward channels at the plasma } \\
\text { membrane }\end{array}$ \\
\hline$K_{\text {out }}$ & $\mathrm{K}^{+}$outward channels at plasma membrane & $L P L$ & lysophospholipids \\
\hline $\mathrm{NADPH}$ & reduced form of nicotinamide adenine dinucleotide phosphate & NIA1 & nitrate reductase \\
\hline NO & nitric oxide & OST1 & protein kinase open stomata 1 \\
\hline PA & phosphatidic acid & PEPC & phosphoenolpyruvate carboxylase \\
\hline phot1 & phototropin 1 & phot $1_{\text {complex }}$ & 14-3-3 protein bound phototropin 1 \\
\hline phot2 & phototropin 2 & $\begin{array}{l}\text { Photophos- } \\
\text { phorylation }\end{array}$ & $\begin{array}{l}\text { light-dependent reactions of } \\
\text { photosynthesis }\end{array}$ \\
\hline $\mathrm{PIP}{ }_{\mathrm{C}}$ & phosphatidylinositol 4,5-bisphosphate located in the cytosol & PIP2PM & $\begin{array}{l}\text { phosphatidylinositol 4,5-bisphosphate } \\
\text { located at the plasma membrane }\end{array}$ \\
\hline$P L A_{2} \beta$ & phospholipase $A 2 \beta$ & PLC & phospholipase C \\
\hline PLD & phospholipase D & PMV & $\begin{array}{l}\text { electric potential difference across the } \\
\text { plasma membrane }\end{array}$ \\
\hline$P P 1_{\mathrm{cn}}$ & the catalytic subunit of type 1 phosphatase located in the nucleus & $\mathrm{PP} 1_{\mathrm{cc}}$ & $\begin{array}{l}\text { the catalytic subunit of type } 1 \text { phosphatase } \\
\text { located in the cytosol }\end{array}$ \\
\hline $\begin{array}{l}\text { protein } \\
\text { kinase }\end{array}$ & $\begin{array}{l}\text { a serine/threonine protein kinase that directly phosphorylates the } \\
\text { plasma membrane H-ATPase }\end{array}$ & PRSL1 & $\begin{array}{l}\text { type } 1 \text { protein phosphatase regulatory } \\
\text { subunit 2-like protein } 1\end{array}$ \\
\hline RIC7 & ROP-interactive CRIB motif-containing protein 7 & $\mathrm{ROP} 2$ & small GTPase ROP2 \\
\hline ROS & reactive oxygen species & {$\left[\mathrm{Ca}^{2+}\right]_{\mathrm{c}}$} & cytosolic $\mathrm{Ca}^{2+}$ concentration \\
\hline$\left[\mathrm{Cl}^{-}\right]_{C / N}$ & cytosolic/vacuolar $\mathrm{Cl}^{-}$concentration & {$\left[\mathrm{K}^{+}\right]_{\mathrm{C} / \mathrm{N}}$} & cytosolic/vacuolar $\mathrm{K}^{+}$concentration \\
\hline$\left[\text { malate }^{2-}\right]_{\mathrm{a} / \mathrm{c} / \mathrm{N}}$ & apoplastic/ cytosolic/vacuolar malate ${ }^{2-}$ concentration & {$\left[\mathrm{NO}_{3}^{-}\right]_{\mathrm{a} / \mathrm{c} / \mathrm{v}}$} & $\begin{array}{l}\text { apoplastic/cytosolic/vacuolar nitrate } \\
\text { concentration }\end{array}$ \\
\hline
\end{tabular}

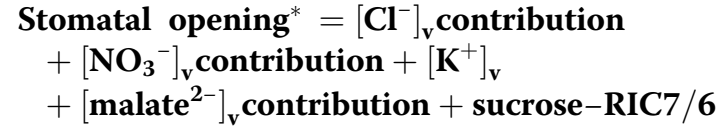

The weights of the anion contributions to the osmotic potential were chosen based on the literature. Also, the anion contributions must not exceed a proportion of $\left[\mathrm{K}^{+}\right]_{\mathrm{v}}$ due to charge balance. The anion contributions are $\left[\text { malate }^{2-}\right]_{\mathrm{v}}$ contribution $\leq 0.425 \times\left[\mathrm{K}^{+}\right]_{\mathrm{v}}$; $\left[\mathrm{NO}_{3}^{-}\right]_{\mathrm{v}}$ contribution $\leq 0.10 \times\left[\mathrm{K}^{+}\right]_{\mathrm{v}} ;\left[\mathrm{Cl}^{-}\right]_{\mathrm{v}}$ contribution $\leq$
$0.05 \times\left[\mathrm{K}^{+}\right]_{\mathrm{v}}$. The primary contributions come from $\left[\mathrm{K}^{+}\right]_{\mathrm{v}}$ and sucrose. We grouped the stomatal opening values into 6 groups with different $\left[\mathrm{K}^{+}\right]_{\mathrm{v}}$ and sucrose values (see Table 2 and Additional file 1).

The first two columns indicate the $\left[\mathrm{K}^{+}\right]_{\mathrm{v}}$ and sucrose levels. The third column is the possible values of stomatal opening in the Sun et al. model for the given $\left[\mathrm{K}^{+}\right]_{\mathrm{v}}$ and sucrose levels. Note that here we only show $\left[\mathrm{K}^{+}\right]_{\mathrm{v}}$, sucrose and stomatal opening value combinations observed in the simulations of the 66 
Table 2 Grouping of the stomatal opening values by the level of $\left[\mathrm{K}^{+}\right]_{V}$ and sucrose

\begin{tabular}{llll}
\hline$\left[\mathrm{K}^{+}\right]_{V}$ & Sucrose & $\begin{array}{l}\text { Stomatal opening value } \\
\text { in the Sun et al. model }\end{array}$ & $\begin{array}{l}\text { Simplified stomatal } \\
\text { opening value }\end{array}$ \\
\hline 0 & 0 & 0 & 0 \\
0 & 1 or 2 & 1 or 2 & 1 \\
1 & 0 & 1.58 & 1 \\
1.8 & 1 & 3.84 & 2 \\
1.5 & 2 & 4.36 & 2 \\
2 & 0 or 1 & 3.15 or 4.15 & 3 \\
4.5 & 0 or 2 & 5.18 or 8.92 & 3 \\
6 & 0 & 9.28 or 9.45 & 5 \\
6 & 2 & 11.28 or 11.45 & 5 \\
9 & 0 or 2 & 14.01 or 16.01 & 6 \\
\hline
\end{tabular}

experimentally studied scenarios reported by Sun et al. [15]. More stomatal opening values are possible when considering node perturbations. The 4th column shows the simplified stomatal opening level after grouping. The update function for the simplified stomatal opening level covers all possible values of $\left[\mathrm{K}^{+}\right]_{\mathrm{v}}$ and sucrose (see Additional file 1).

Similarly to the original model, the simplified states represent qualitative, relative categories. For example, a stomatal opening level of 2 is not twice as high as level 1 . We choose the simplified stomatal opening values so that there is no state " 4 ", to better reflect an experimentally observed synergistic effect between blue and red light $[18,19,27]$. Simulation results with the simplified regulatory function are that under monochromatic red light stomatal opening $=1$; under monochromatic blue light stomatal opening $=3$; under dual beam the stomatal opening $=5$, which is larger than the sum " $1+3$ ". This qualitatively reproduces the experimental observation that under dual beam illumination stomata open to a size much larger than the sum of opening under monochromatic blue or red light.

We find by simulation of the reduced model, using the same initial condition as the Sun et al. model, that the simplification of the stomatal opening regulatory function results in only 3 additional cases of inconsistency with experimental observations out of a total of 66 experimentally studied scenarios. Additional file 2 lists all experimental observations and compares them to the relevant simulation results. Ignoring the contribution of malate ${ }^{2-}, \mathrm{NO}_{3}^{-}$, and RIC7 to stomatal opening each causes one additional discrepancy; ignoring $\mathrm{Cl}^{-}$ does not cause any additional discrepancy. Ignoring these nodes trades a decrease in accuracy for a significant increase in simplicity.

The simplification of the stomatal opening regulatory function eliminates the effect of vacuolar anions and of
RIC7 on stomatal opening. As a result we can further simplify the Sun et al. model by eliminating 10 nodes in total, $\left[\text { malate }^{2-}\right]_{\mathrm{a}}, \quad\left[\text { malate }{ }^{2-}\right]_{\mathrm{c}}$, starch, $\left[\mathrm{Cl}^{-}\right]_{\mathrm{c}},\left[\mathrm{NO}_{3}^{-}\right]_{\mathrm{c}}$, $\left[\mathrm{NO}_{3}^{-}\right]_{\mathrm{a}}, \mathrm{ROP} 2, \mathrm{RIC7}, \mathrm{ABC}$, and PEPC. The only edge from these nodes to other nodes is $\left[\text { malate }^{2-}\right]_{\mathrm{a}} \rightarrow$ AnionCh. In section 3 of Additional file 3 we show that eliminating this edge does not change the system's longterm behavior, i.e. attractors. Also, the regulatory function describing the cytosolic $\mathrm{K}^{+}$concentration, $\left[\mathrm{K}^{+}\right]_{\mathrm{c}}$, can be simplified without loss, as described in section 3 of Additional file 3. After this simplification we have a network of 32 nodes, 81 edges, indicated on Fig. 2. We will refer to this model as the "reduced model". A list of nodes and their regulatory functions is provided in Additional file 1.

Identifying strongly connected components (SCCs) is important for attractor analysis, as complex dynamic behavior such as oscillations or multi-stability requires feedback loops [7]. There are three SCCs in the network of the reduced model, as marked in Fig. 2. The NO cycle contains three nodes and three positive edges. The $\mathrm{C}_{\mathrm{i}} \mathrm{SCC}$ contains three nodes, which form two negative feedback loops. The Ion SCC is the most complex, containing 13 nodes and 26 edges, 7 of which are negative.

Next we perform attractor analysis using two methods: 1 . by converting the reduced model to Boolean and applying two analysis tools; 2 . by analyzing the regulatory functions theoretically. The former method finds all stable steady states and candidate oscillations; the latter confirms the results of the first method and gives insight about perturbation scenarios.

\section{Conversion of nodes from multi-level to Boolean states and attractor analysis}

We perform the conversion to Boolean to enable attractor analysis by existing software tools. Zañudo et al. [28] proposed an algorithm to find the attractors of a Boolean network based on the concept of "stable motif", a strongly-connected group of nodes that can stabilize regardless of their inputs. The algorithm finds all stable motifs, which determine the part of the network that stabilizes in an attractor. After a stable motif is found, one can plug in its stabilized state into the network, and obtain a smaller remaining network. After repeating this, eventually the remaining part is either nothing (indicating a fixed point/stable steady state) or a candidate oscillating sub-network. Compared with other software tools $[29,30]$, the major advantage of this algorithm is that it finds all the attractors of Boolean networks with hundreds of nodes [28]. Application of this powerful method requires a Boolean model, so we convert the multi-level model into Boolean first (see Methods). An example of conversion is given in Table 3 . 


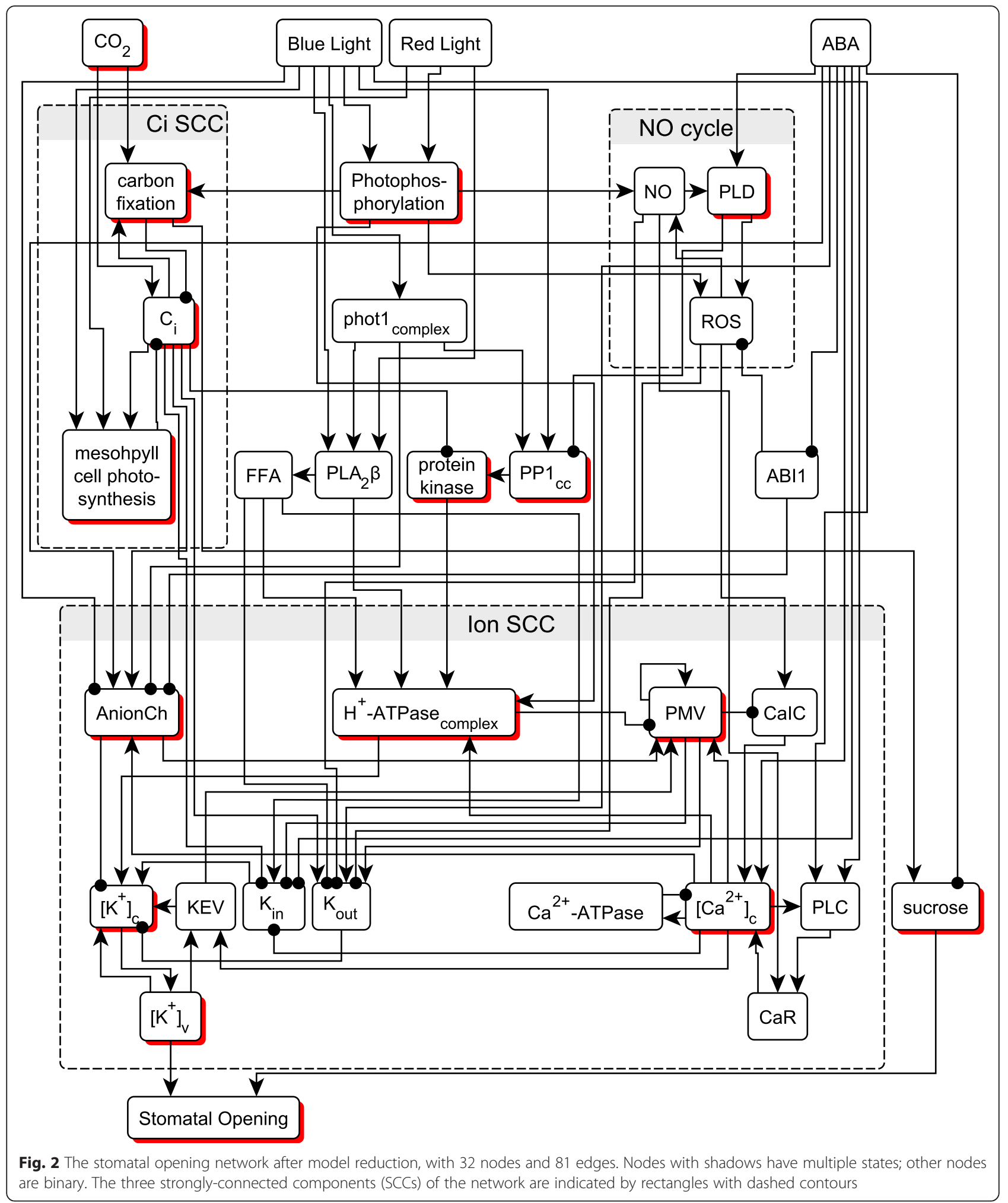

More detailed examples of the conversion of the states and regulatory function of specific nodes are given in the Additional file 4. We will refer to the reduced model after conversion to Boolean variables as the "Boolean- converted reduced model". The regulatory functions of the Boolean-converted reduced model are available in Additional file 5. When simulating the Booleanconverted reduced model, all the Boolean nodes that 
Table 3 Example of Boolean conversion

\begin{tabular}{lll}
\hline $\begin{array}{l}\text { Level of the original } \\
\text { node }\end{array}$ & $\begin{array}{l}\text { State of Boolean } \\
\text { node_2 }\end{array}$ & $\begin{array}{l}\text { State of Boolean } \\
\text { node_1 }\end{array}$ \\
\hline 0 & 0 & 0 \\
1 & 0 & 1 \\
2 & 1 & 0 \\
3 & 1 & 1 \\
\hline
\end{tabular}

The multi-level node shown in the 1st column is mapped into two Boolean nodes, shown in the 2 nd and 3rd columns, using the binary representation of the corresponding integer.

represent the same entity (the same multi-level node) are updated simultaneously. In this way the state transitions of the reduced model will be kept the same in the Boolean-converted reduced model, and therefore the Boolean conversion will not cause additional discrepancies from experimental observations.

We apply the stable motif algorithm's implementation, downloaded from http://github.com/jgtz/StableMotifs/ [28], to the Boolean-converted reduced model. The algorithm uses the Boolean regulatory functions of the converted model (given in Additional files 5 and 6) as input. We consider every combination of sustained states of the five signal nodes (blue light, red light, $\mathrm{ABA}, \mathrm{CO}_{2}$, $\mathrm{CO}_{2}$ high). We find two possible stable motifs, corresponding to the self-regulatory node PMV_pos (one of the two Boolean nodes associated with the multi-level node PMV, see Additional files 4 and 5), in conditions where the $\mathrm{H}^{+}$-ATPase complex $_{\text {is inactive. These two stable }}$ motifs indicate the bistability of PMV. Under its influence, another node, $K_{\text {out }}$, will also be bistable. The algorithm also indicates that for any signal combination, every node, except $\left[\mathrm{Ca}^{2+}\right]_{\mathrm{c}}$ and $\mathrm{Ca}^{2+}$-ATPase, will stabilize in a fixed state. $\left[\mathrm{Ca}^{2+}\right]_{\mathrm{c}}$ has three states, and in the Boolean-converted model it is represented by two nodes, Cac and Cac_high. Cac_high, which represents the higher level of $\left[\mathrm{Ca}^{2+}\right]_{\mathrm{c}}$, stabilizes at zero in all situations. Cac and $\mathrm{Ca}^{2+}$-ATPase may oscillate in conditions where blue light is present and ABA is absent (a total of six cases, two of which allow PMV bistability). Table 4 summarizes key features of the attractors found by the stable motif algorithm for all 24 input combinations. Attractors where $\mathrm{Ca}^{2+}$ oscillation is not possible are fixed points (stable steady states).

We verified the obtained attractors with GINsim [12], a software suite capable of model construction, simulation, and analysis. GINsim can compute all stable steady states (called stable states in GINsim), or determine complex attractors by mapping the state transitions. The stable steady states found by GINsim are identical to those found by the stable motif algorithm. To verify and further explore the complex attractors, we use the simulation function of GINsim, starting from a state in the complex attractor. The result that the system oscillates between four states, where only the state of $\mathrm{Cac}$ and $\mathrm{Ca}^{2}$ ${ }^{+}$-ATPase changes, agrees with the findings of the stable motif algorithm. We summarize the GINsim computation/simulation results in Additional file 7. Additional file 8 indicates the Boolean-converted reduced model in SBML-qual format [29], a general format for biological model to be analyzed using various tools including GINsim.

We can also connect the stable motif analysis results to network reduction. We have previously decided to not reduce the four nodes that correspond to input signals. If we do consider a specific input combination when using network reduction, e.g. blue light and red light with normal $\mathrm{CO}_{2}$ without $\mathrm{ABA}$, we can reduce much more of the network: two of the three SCCs, namely the $\mathrm{NO}$ cycle and the $\mathrm{C}_{\mathrm{i}} \mathrm{SCC}$, will stabilize and can be eliminated. Only the Ion SCC and its sole output stomatal opening remain, indicating that this SCC is not driven solely by the external signals and has the capacity for oscillations or multi-stability. This is consistent with the results found by stable motif analysis, according to which the $\mathrm{NO}$ cycle and the $\mathrm{C}_{\mathrm{i}} \mathrm{SCC}$ attain a steady state and the Ion SCC admits a $\left[\mathrm{Ca}^{2+}\right]_{\mathrm{C}}-\mathrm{Ca}^{2+}$-ATPase oscillation and PMV bistability. This consistency supports the appropriateness of the network reduction method and of the Boolean conversion.

\section{Theoretical analysis of the reduced model}

To gain additional insight into the attractors of the reduced model and their potential changes due to node perturbations, we analyze the reduced model theoretically. Specifically, we aim to answer the question: Can there be other types of oscillation, or can there be additional multi-stability, if a node is knocked out (fixed in the OFF state) or is constitutive active (fixed in the highest state)?

We first test whether the network and regulatory rules allow multi-stability or oscillations. This analysis is based on R. Thomas's conjectures [7]: The presence of a positive (negative) feedback loop - a cycle with an even (odd) number of inhibitory edges - in the network is a necessary but not sufficient condition for the occurrence of multiple steady states (oscillations). The conjectures have been proven in the case of discrete dynamic systems [31-34]. Since only feedback loops are candidates for potential multi-stability or oscillations, we analyze the regulatory functions of each strongly connected component of the network. For each feedback loop, we identify a sufficient condition for the nodes to stabilize in a specific state. The violation of this condition becomes a further necessary condition of multi-stability or oscillation. Here we describe the main steps and results of the analysis; the detailed analysis is in Additional file 3. 
Table 4 Summary of the attractors found using the stable motif algorithm

\begin{tabular}{lllllllll}
\hline $\mathrm{BL}$ & $\mathrm{RL}$ & $\mathrm{CO}_{2}$ & $\mathrm{CO}_{2}$ high & $\mathrm{ABA}$ & $\mathrm{SO}$ (Bool) & $\mathrm{SO}$ & $\mathrm{Ca}^{2+}$ Oscillation Possible? & PMV_pos bistability \\
\hline 0 & 0 & Any & Any & Any & 000 & 0 & No & Nes \\
0 & 1 & 0 & 0 & 1 & 000 & 0 & No & Yes \\
0 & 1 & 1 & Any & 1 & 000 & 0 & No & No \\
1 & Any & 1 & 0 & 1 & 000 & 0 & No & Yes \\
1 & Any & 1 & 1 & 1 & 000 & 0 & No & Yes \\
0 & 1 & 1 & Any & 0 & 010 & 1 & No & Yes \\
1 & Any & 1 & 1 & 0 & 010 & 1 & Yes & No \\
0 & 1 & 0 & 0 & 0 & 101 & 3 & No & No \\
1 & 0 & 1 & 0 & 0 & 101 & 3 & Yes & No \\
1 & Any & 0 & 0 & 1 & 101 & 3 & No & No \\
1 & 0 & 0 & 0 & 0 & 110 & 5 & Yes & No \\
1 & 1 & 1 & 0 & 0 & 110 & 5 & Yes & No \\
\hline
\end{tabular}

The first five columns indicate the input signal combination. The setting $\mathrm{CO}_{2}$ high $=1$ and $\mathrm{CO}_{2}=0$ is not included because it is not biologically meaningful. The "SO (Bool)" column indicates the state of the Boolean node combination representing stomatal opening. The "SO" column is the state of stomatal opening when converted back to an integer. Note that the stomatal opening level of four is not defined, and no attractors have a stomatal opening level of two. The next column indicates whether $\mathrm{Ca}^{2+}$ oscillation can possibly happen under the given signal combination. The last column indicates whether bistability of PMV_pos can be observed under this setting. In those cases, two stable steady states with (PMV_pos $\left.=0, K_{\text {out }}=0\right)$ and $\left(P M V \_p o s=1, K_{\text {out }}=1\right)$ can be observed. The rest of the nodes are unaffected by this two-node bistability

The NO cycle is composed of the nodes PLD, ROS, $\mathrm{NO}$, and the three positive edges between them. It does not have any negative edges, so it cannot oscillate. A fixed ABA value is sufficient to stabilize each node of the cycle in a specific state, thus the cycle does not admit multi-stability under any perturbation.

The $C_{i}$ SCC has three nodes, $C_{i}$, mesophyll cell photosynthesis (MCPS), carbon fixation, and four edges that form two negative feedback loops, one between carbon fixation and $C_{i}$, and the other between $C_{i}$ and MCPS. Despite the existence of negative feedback, this cycle will stabilize if given a fixed $\mathrm{CO}_{2}$ value. From this we know that this cycle cannot oscillate or admit multi-stability under any perturbation.

The Ion SCC has 13 nodes. To reduce its complexity we show that the key node $\left[\mathrm{Ca}^{2+}\right]_{\mathrm{c}}$, which has states 0,1 , and 2, cannot enter state 2 in the long term under any perturbation. Since most nodes respond to $\left[\mathrm{Ca}^{2+}\right]_{\mathrm{c}}$ only if $\left[\mathrm{Ca}^{2+}\right]_{\mathrm{c}}=2$, we can eliminate all edges that depend only on " $\left[\mathrm{Ca}^{2+}\right]_{\mathrm{c}}=2$ ", and obtain a simplified Ion SCC, as shown in Fig. 3. The $\mathrm{Ca}^{2+} \mathrm{SCC}\left(\left[\mathrm{Ca}^{2+}\right]_{\mathrm{C}}, \mathrm{Ca}^{2+}\right.$ ATPase, PLC, CaR) now becomes a sink SCC. The only negative edge in this sub-network is from $\mathrm{Ca}^{2+}$-ATPase to $\left[\mathrm{Ca}^{2+}\right]_{\mathrm{C}}$. These two nodes are known to oscillate. The positive feedback loop formed by $\left[\mathrm{Ca}^{2+}\right]_{\mathrm{c}}, \mathrm{PLC}$, and CaR will stabilize if given fixed inputs. So there cannot be multi-stability. For the nodes outside of the $\mathrm{Ca}^{2+}$ feedback loops, we show that the edges from KEV and $\left[\mathrm{K}^{+}\right]_{\mathrm{V}}$ are redundant in the long term, so there are no feedback loops except the PMV selfloop. PMV is not capable of having oscillations, but can have bistability (as also indicated by the stable motif analysis). The bistability can affect at most one other node, $K_{\text {out }}$, under any perturbation. This means that the bistability has very limited effect on the attractor of the reduced model.

Now we can summarize our conclusions and return to the question we sought to answer: there is no oscillation except in the calcium nodes; there is no multi-stability except in the nodes PMV and $K_{\text {out }}$. These statements are true under any perturbation. Moreover, for the calcium oscillation, $\left[\mathrm{Ca}^{2+}\right]_{\mathrm{c}}$ cannot enter the state 2 , so the subnetwork between $\left[\mathrm{Ca}^{2+}\right]_{\mathrm{c}}$ and $\mathrm{Ca}^{2+}$-ATPase is a negative feedback loop between two Boolean nodes, with the regulatory functions $\mathrm{Ca}^{2+}$ ATPase $=\left[\mathrm{Ca}^{2+}\right]_{\mathrm{c}} ;\left[\mathrm{Ca}^{2+}\right]_{c}{ }^{*}=$ not $\mathrm{Ca}^{2+}$ ATPase. It results in the simplest type of oscillation, as also found by GINsim simulation. For the PMV bistability, even if the bistability exists, most nodes, especially the output node stomatal opening, still have a unique value. Thus the theoretical analysis, in agreement with the computational analysis, leads to very strong conclusions about the reduced model's dynamic repertoire.

We can also show that the reduction or Boolean conversion did not change the attractors of the Sun et al. model. Although the reduction we used is only proven in the Boolean case, Naldi et al. showed that for multivalued models, removal of non-autoregulated nodes, like in our reduction, preserves crucial dynamical properties [35], including fixed point attractors and the two-node simple oscillation we found. So our reduction is valid in this specific model. To confirm that the Boolean conversion preserved attractors, we note that in the Boolean- 


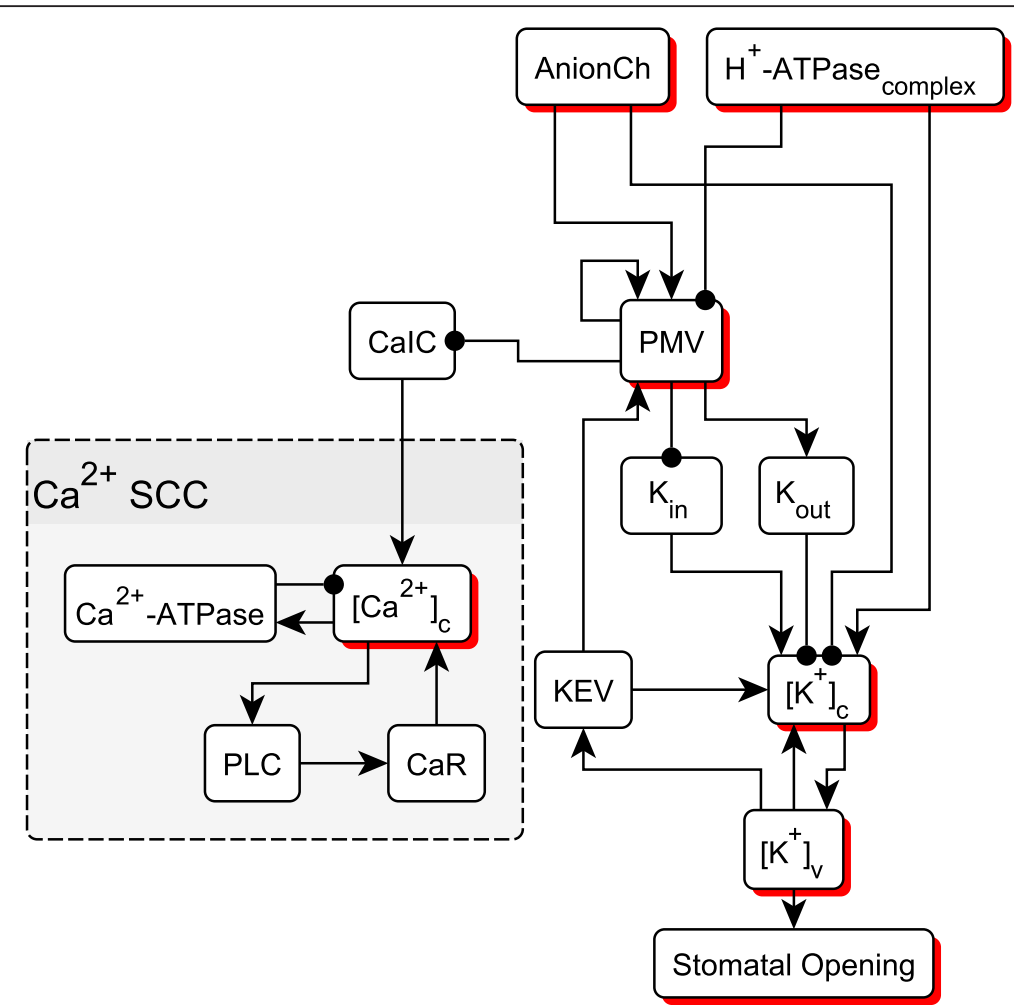

Fig. 3 The Ion SCC after reducing all edges that depend on calcium. All regulators of this sub-network have been omitted. On the left, [Ca $\left.{ }^{2+}\right]_{c}$ related nodes form a sink sub-network

converted reduced model we found fixed point attractors and a complex attractor in which only two nodes oscillate. Because the only potential change to attractors as a consequence of the conversion is merging of complex attractors [26], it is straightforward that the attractors have been conserved during the conversion, as the twonode oscillation found is the simplest type of complex attractor and cannot be a result of attractor merging. In addition, using general asynchronous update instead of random order asynchronous update does not cause any changes to the attractor, because the update schemes do not affect fixed points or the two-node simple oscillation we found.

\section{Stability of guard cell signal transduction}

Our previous results indicate the stability of the system in the sense that all the initial conditions lead to the same attractor except for up to four nodes. We also examine another facet of the system's stability: the robustness of the stomatal opening in response to node perturbations that render them non-functional. We perform a systematic analysis of single-node knockouts of every non-signal node in the reduced model, under all combinations of light, $\mathrm{CO}_{2}$ and $\mathrm{ABA}$ conditions. For each signal combination, we set the perturbed node's initial state and regulatory function to 0 , initialize the rest of the nodes in the condition representative of closed stomata, and then simulate the reduced model until it reaches its attractor. In the absence of ABA under each light and $\mathrm{CO}_{2}$ condition, 60-90\% perturbation scenarios produce the same stomatal opening value as the unperturbed system (Table 5). These results are similar to those reported by Sun et al. for the original model [15] (see Additional file 9). In the presence of ABA 50-90\% perturbation scenarios produce the same stomatal opening value as the unperturbed system, and 4-16\% knockouts lead to a higher stomatal opening value. Perturbations in the ABA $=1$ case were not studied by Sun et al., but our simulations of the original model give the same qualitative results as the reduced model. These results indicate the closeness of the perturbed attractor (at least in terms of the stomatal opening value) to the unperturbed attractor in more than $50 \%$ of single node perturbations. They also suggest the resilience of the stomatal opening process against internal failures and perturbations.

\section{Extending the conclusions to the original model}

We found that in the reduced model there is no oscillation except in the calcium nodes; there is no multistability except in the nodes PMV and $\mathrm{K}_{\text {out }}$. Because the reduction we used has been shown to conserve 
Table 5 Summary of systematic perturbation results

\begin{tabular}{|c|c|c|c|c|c|c|c|c|c|c|}
\hline \multirow{2}{*}{\multicolumn{3}{|c|}{ Light, $\mathrm{CO}_{2}$ and $\mathrm{ABA}$ condition }} & \multirow{4}{*}{$\begin{array}{l}\text { Unperturbed } \\
\text { SO level } \\
\\
5\end{array}$} & \multirow{2}{*}{\multicolumn{6}{|c|}{ Simplified SO level }} & \multirow{3}{*}{$\begin{array}{l}\text { Percentage } \\
\text { of cases } \\
\text { with } \\
\text { unchanged } \\
\text { SO value } \\
65 \%\end{array}$} \\
\hline & & & & & & & & & & \\
\hline & & & & \multicolumn{6}{|c|}{ Percentage of single knockouts that lead to each SO level } & \\
\hline \multirow[t]{3}{*}{ Dual Beam } & Mod. $\mathrm{CO}_{2}$ & ABA OFF & & & $4 \%$ & & $31 \%$ & $65 \%$ & & \\
\hline & Low $\mathrm{CO}_{2}$ & & 6 & $31 \%$ & & & $4 \%$ & & $65 \%$ & $65 \%$ \\
\hline & High $\mathrm{CO}_{2}$ & & 1 & $4 \%$ & $96 \%$ & & & & & $96 \%$ \\
\hline \multirow[t]{3}{*}{ Blue Light } & Mod. $\mathrm{CO}_{2}$ & & 3 & & $35 \%$ & & $65 \%$ & & & $65 \%$ \\
\hline & Low $\mathrm{CO}_{2}$ & & 5 & $31 \%$ & & & $4 \%$ & $65 \%$ & & $65 \%$ \\
\hline & High $\mathrm{CO}_{2}$ & & 1 & $4 \%$ & $96 \%$ & & & & & $96 \%$ \\
\hline \multirow[t]{3}{*}{ Red Light } & Mod. $\mathrm{CO}_{2}$ & & 1 & $4 \%$ & $96 \%$ & & & & & $96 \%$ \\
\hline & Low $\mathrm{CO}_{2}$ & & 3 & $35 \%$ & & & $65 \%$ & & & $65 \%$ \\
\hline & $\mathrm{High} \mathrm{CO}_{2}$ & & 1 & $4 \%$ & $96 \%$ & & & & & $96 \%$ \\
\hline \multirow[t]{2}{*}{ Dual Beam } & Mod. $\mathrm{CO}_{2}$ & ABA ON & 0 & $85 \%$ & & $4 \%$ & $8 \%$ & $4 \%$ & & $85 \%$ \\
\hline & Low $\mathrm{CO}_{2}$ & & 3 & $46 \%$ & & & $50 \%$ & & $4 \%$ & $50 \%$ \\
\hline \multirow[t]{2}{*}{ Blue Light } & Mod. $\mathrm{CO}_{2}$ & & 0 & $85 \%$ & $4 \%$ & $8 \%$ & $4 \%$ & & & $85 \%$ \\
\hline & Low $\mathrm{CO}_{2}$ & & 3 & $46 \%$ & & & $50 \%$ & $4 \%$ & & $50 \%$ \\
\hline Red Light & Low $\mathrm{CO}_{2}$ & & 0 & $96 \%$ & & & $4 \%$ & & & $96 \%$ \\
\hline
\end{tabular}

The first set of columns, with the header 'Light, $\mathrm{CO}_{2}$ and $\mathrm{ABA}$ condition', indicate the input signal combinations. The abbreviation "Mod." means moderate $\mathrm{CO}_{2}$ concentration. Note that we do not list the four input combinations (high $\mathrm{CO}_{2}$ with $\mathrm{ABA}$ and with any type of light, or moderate $\mathrm{CO}_{2}$ with $\mathrm{ABA}$ and red light) wherein all simulated stomatal opening values are zero. The 2 nd column is the simulated stomatal opening (SO) level in the unperturbed system. The 3 rd column set shows the percentage of single-node knockouts that yield the corresponding SO level. There is no stomatal opening level 4 in the reduced model. No entry means zero percentage. The last column is the percentage of settings where the stomatal opening remains at the same level as the unperturbed case. A complete table of perturbation results is provided in Additional file 9

attractors [23, 35], we know that our attractor conclusions can be immediately extended to all nodes in the original model except the reduced nodes and stomatal opening. Next we extend the attractor analysis to include the reduced nodes as well.

First we consider the nodes reduced during the first step of network reduction, i.e. non-signal source nodes and simple mediator nodes. These nodes are trivially incapable of having multi-stability and oscillations themselves, so we need only to consider their perturbations. Perturbation of a simple mediator node can always be replaced by a corresponding (set of) perturbation(s) in the mediator node's direct successor(s), so these perturbations have already been considered. Perturbing a nonsignal source node may theoretically cause a difference, however the nodes in this category in the Sun et al. model represent molecules that are abundant in the cell or cell environment, thus their perturbation is not biologically relevant or practical.

Next we consider the anion nodes reduced due to the simplified stomatal opening rule. Recall that these nodes do not affect other nodes except stomatal opening in the long term. There cannot be multi-stability in anion nodes unless the assumptions of sufficient initial $\left[\mathrm{NO}_{3}^{-}\right]_{\mathrm{a}}$ and starch concentration, and sufficient initial mitochondrial TCA cycle activity are violated (details are provided in Additional file 3, section 5 and 6). Since there is no support for interventions that would lead to the violation of these assumptions, it is reasonable to conclude that no multi-stability can be found in the reduced nodes under biologically relevant situations. We also found that there can be an additional oscillation in the RIC7 path (involving the nodes ROP2, RIC7 and SO) when a special set of perturbations is applied. Under that case, the nodes RIC7 and SO will oscillate. Since the effect of this behavior is small (within $5 \%$ of the unperturbed SO value in the Sun et al. model [15]), it has little biological significance. There are no more possible oscillations as there are no more negative feedback loops. To conclude, the original Sun et al. model has oscillations only in cytosolic $\mathrm{Ca}^{2+}\left(\left[\mathrm{Ca}^{2+}\right]_{\mathrm{c}}\right)$ and $\mathrm{Ca}^{2+}$ ATPase, and has multi-stability only in PMV and $\mathrm{K}_{\text {out }}$, under situations that are biologically meaningful.

\section{Discussion}

The conclusions we obtained can tell us how to control this network model. Generally in engineering applications, control means to drive a system into an arbitrary state [36, 37]. However in biological systems, it is more meaningful to drive the system into one of its natural attractors rather than into an arbitrary state, as the attractors correspond to stable phenotypes [38]. To control the attractor of a Boolean system, one needs to control only its input nodes and a subset of nodes in each 
stable motif [39]. Our integrated analysis, involving Boolean conversion, indicates that to control the attractor that the stomatal opening network evolves into, one only needs to control the input signals and PMV, even in case of perturbations. In particular, to control the stomatal opening value, one only needs to control the input signals, under any perturbation.

The reduced model provides new biological insights. Normally, when ABA is present, stomata will close. However in some knockout mutants stomata can open to a certain extent in the presence of $\mathrm{ABA}$, although the opening level is not as much as in the case without ABA [15]. Such partial reversals of the effect of $\mathrm{ABA}$ are important for understanding the mechanism of stomatal opening. For example, Sun et al. reported that OST1 knockout (OST1 is kept 0) and inhibition of the NADPH oxidase (AtrbohD/F is kept 0 ) yielded partially restored SO level in simulations, in agreement with experimental observations (see Additional file 2 for the comparison of the equivalent simulations in the reduced model with experiments). Simplification of the Sun et al. model allows easier simulation of more perturbation scenarios, e.g. the systematic identification of possible partial reversals. Table 6 indicates all the partial reversals due to single node knockouts in the reduced model.

Our results reproduce the observation that knockout of nodes in the ABA pathway (PLD, NO, ROS) can cause partial reversals of ABA's effect. We find that AnionCh knockout can partially restore stomatal opening inhibited by ABA, a result not reported by Sun et al., but which is supported by experimental evidence [40]. In addition, Table 6 offers a new biological prediction: low $\mathrm{CO}_{2}$ concentration can partially restore stomatal opening when $\mathrm{ABA}$ is present. This is consistent with the knowledge that $\mathrm{CO}_{2}$-free air promotes stomatal opening in the absence of $\mathrm{ABA}$ [41]. This $\mathrm{CO}_{2}$ effect suggests a mechanism of cross-talk between $\mathrm{CO}_{2}$ and $\mathrm{ABA}$. Importantly, apart from the five nodes listed in Table 6, no other node's knockout can reverse ABA's inhibition of stomatal opening. The perturbation results of Table 5 offer many more new predictions.

Our combination of techniques offers a powerful framework for determining the dynamic repertoire of a multi- level dynamic model. Multi-level models are more accurate than Boolean models in describing the quantitative characteristics of dynamic systems, but there are few general methods to analyze multi-level models $[10,12]$. By combining different existing methods, we were able to overcome the limitations of each method. Our successful combination of existing methods offers a promising way to analyze multi-level models, and might point towards a general strategy to analyze the attractors of multi-level models, biological or non-biological.

A notable future direction for this work is to develop an alternative way to determine the attractors of multilevel models by extending the concept of stable motifs. Compared with conversion to a Boolean model, then applying Boolean stable motif algorithm, extending the stable motif algorithm to multi-level models can avoid potential attractor change issues. Development of such a technique will allow easy and powerful attractor analysis for multi-level models.

\section{Conclusions}

We obtained a very strong conclusion about the attractors of the Sun et al. stomatal opening model: under any combination of sustained signals, all nodes in the model converge into steady states, with the potential exception of the cytosolic $\mathrm{Ca}^{2+}\left(\left[\mathrm{Ca}^{2+}\right]_{\mathrm{c}}\right)$ and $\mathrm{Ca}^{2+}$ ATPase. Variations in the initial condition of non-source nodes or in process timing (node update sequence) can drive at most two nodes, PMV and $\mathrm{K}_{\text {out }}$, into a different attractor. This high degree of attractor similarity is somewhat unexpected, as the network has a large strongly connected component and several feedback loops. Thus, despite the decidedly non-linear structure of the network, most parts of the system behave in the consistent manner of a linear pathway. This is a distinct feature of the stomatal opening model: many dynamic models of biological systems have multiple, diverse attractors [22, 42]. The models of these systems will evolve into drastically different attractors when starting from different initial conditions, sometimes even when starting from the same initial condition, demonstrating different biological trajectories. In the stomatal opening model, however, the uniqueness of the steady state stomatal opening level suggests that the final extent of the stomatal opening response is robust and resilient

Table 6 Nodes whose knockouts diminish ABA's inhibition of stomatal opening

\begin{tabular}{|c|c|c|c|c|c|c|}
\hline \multirow[t]{2}{*}{ Light, $\mathrm{CO}_{2}$ and $\mathrm{ABA}$ condition } & \multirow{2}{*}{$\begin{array}{l}\text { Unperturbed } \\
\text { SO level }\end{array}$} & \multicolumn{5}{|c|}{ Nodes whose knockout results in a partially restored SO, and the corresponding SO value } \\
\hline & & $\mathrm{CO}_{2}$ & NO & PLD & ROS & AnionCh \\
\hline Dual Beam & 0 & 3 & 3 & 5 & 3 & 2 \\
\hline Blue Light Moderate $\mathrm{CO}_{2}, \mathrm{ABA}$ is present & 0 & 3 & 2 & 3 & 2 & 1 \\
\hline Red Light & 0 & & & 3 & & 1 \\
\hline
\end{tabular}

The first set of columns, with the header 'Light, $\mathrm{CO}_{2}$ and ABA condition', indicate the input signal combinations. The 2nd column is the stomatal opening without perturbations. The $3 \mathrm{rd}$ column set indicates the nodes whose knockout would yield a stomatal opening level that is higher than the unperturbed value of 0 . $\mathrm{CO}_{2}$ knockout means $\mathrm{CO}_{2}$ being set to zero $\left(\mathrm{CO}_{2}\right.$ free air). No entry means the setting does not cause partial reversal 
against changes in initial conditions or in timing. Note that although a change in the initial condition will not change the steady-state opening level, it may change the steady state of PMV and $\mathrm{K}_{\text {out }}$, and may change how fast the system converges to an attractor.

We also showed that the reduced stomatal opening model does not admit additional, emergent oscillations or multi-stability under any biologically relevant node perturbation (knockout or constitutive activity). We further demonstrate the robustness of the system by examining the stomatal opening level under single node knockouts: in most cases the signals are still likely to propagate and lead to a similar degree of stomatal opening as in the absence of perturbation. This robustness is unlike a single linear pathway, which would be very sensitive to node disruption. We suggest that the role of the strongly connected components in the network could be to provide multiple paths for the signal to propagate, but at the same time not allowing extensive multistability or oscillations. Our innovative combination of existing methods offers a promising way to analyze multi-level models.

\section{Additional files}

Additional file 1: Regulatory functions of the reduced stomatal opening model. (DOCX $38 \mathrm{~kb}$ )

Additional file 2: Compilation of comparisons between published experimental observations and the reduced model's results for simulations of the identical conditions. (DOCX $163 \mathrm{~kb}$ )

Additional file 3: Analysis of stomatal opening model. Detailed derivation of attractor analysis and other statements made in the main article. (DOCX $98 \mathrm{~kb}$ )

Additional file 4: Examples of converting a multi-level update function to Boolean. (DOCX $38 \mathrm{~kb}$ )

Additional file 5: Regulatory functions of the Boolean-converted reduced stomatal opening model. (DOCX $25 \mathrm{~kb}$ )

Additional file 6: Text file of the Boolean-converted reduced stomatal opening model to be used in the stable motif algorithm. The stable motif algorithm and instructions about how to use it can be found from this link: https://github.com/jgtz/StableMotifs. (TXT 4 kb)

Additional file 7: GINsim attractor analysis of the Boolean-converted reduced stomatal opening model. (DOCX $42 \mathrm{~kb}$ )

Additional file 8: Boolean-converted reduced stomatal opening model in SBML format. This file format can be used in various tools, including GINsim. (SBML $199 \mathrm{~kb}$ )

Additional file 9: Stomatal opening levels for simulated single node knockouts in the simplified model under all input signal combinations. (XLSX $15 \mathrm{~kb})$

\section{Acknowledgements}

The authors thank Zhongyao Sun, Jorge G. T. Zañudo and Prof. Sarah Assmann for helpful discussions.

\section{Funding}

This project was supported by National Science Foundation (NSF) grants IIS 1160995, PHY 1205840 and MCB 1244303. The NSF had no role in the design of the study, analysis and interpretation of data or in writing the manuscript.

\section{Availability of data and materials}

The datasets supporting the conclusions of this article are included within the article and its additional files.

\section{Authors' contributions}

XG developed the reduced model and performed the analysis under the advice and supervision of RA. Both authors wrote the manuscript. Both authors have read and approved the final version of the manuscript.

\section{Competing interests}

The authors declare that they have no competing interests.

\section{Consent for publication}

Not applicable. The article does not contain any individual person's data.

Ethics approval and consent to participate

Not applicable. The article does not involve human participants, human data or human tissue.

Received: 10 June 2016 Accepted: 11 August 2016

Published online: 19 August 2016

\section{References}

1. Stigler B, Chamberlin HM. A regulatory network modeled from wild-type gene expression data guides functional predictions in Caenorhabditis elegans development. BMC Syst Biol. 2012;6:77.

2. Chifman J, et al. The core control system of intracellular iron homeostasis: a mathematical model. J Theor Biol. 2012;300:91-9.

3. Massague J. TGF-beta signal transduction. Annu Rev Biochem. 1998;67:753-91.

4. $\mathrm{Xu} \mathrm{HL}$, et al. Construction and Validation of a Regulatory Network for Pluripotency and Self-Renewal of Mouse Embryonic Stem Cells. PLoS Comput Biol. 2014;10(8):e1003777.

5. Kestler HA, et al. Network modeling of signal transduction: establishing the global view. Bioessays. 2008:30(11-12):1110-25.

6. Tyson JJ, Chen K, Novak B. Network dynamics and cell physiology. Nat Rev Mol Cell Biol. 2001;2(12):908-16.

7. Thomas R, European Molecular Biology Organization. Kinetic logic : a Boolean approach to the analysis of complex regulatory systems: proceedings of the EMBO course "Formal analysis of genetic regulation," held in Brussels, September 6-16, 1977, Lecture notes in biomathematics. Berlin; New York: Springer; 1979. p. xiii, 507.

8. Kauffman SA. Metabolic stability and epigenesis in randomly constructed genetic nets. J Theor Biol. 1969:22(3):437.

9. Glass L, Kauffman SA. Logical analysis of continuous, nonlinear biochemical control networks. J Theor Biol. 1973;39(1):103-29.

10. Wang RS, Saadatpour A, Albert R. Boolean modeling in systems biology: an overview of methodology and applications. Phys Biol. 2012:9(5):055001.

11. Miskov-Zivanov N, et al. The duration of T cell stimulation is a critical determinant of cell fate and plasticity. Sci Signal. 2013;6(300):ra97.

12. Chaouiya C, Naldi A, Thieffry D. Logical modelling of gene regulatory networks with GINsim. Methods Mol Biol. 2012;804:463-79.

13. Deritei $\mathrm{D}$, et al. Principles of dynamical modularity in biological regulatory networks. Sci Rep. 2016;6:21957.

14. Murrugarra $\mathrm{D}$, Laubenbacher R. Regulatory patterns in molecular interaction networks. J Theor Biol. 2011;288:66-72.

15. Sun $Z$, et al. Multi-level modeling of light-induced stomatal opening offers new insights into its regulation by drought. PLoS Comput Biol. 2014:10(11):e1003930.

16. Li S, Assmann SM, Albert R. Predicting essential components of signal transduction networks: a dynamic model of guard cell abscisic acid signaling. PLoS Biol. 2006;4(10):e312.

17. Schroeder Jl, et al. Guard Cell Signal Transduction. Annu Rev Plant Physiol Plant Mol Biol. 2001:52:627-58.

18. Shimazaki $\mathrm{K}$, et al. Light regulation of stomatal movement. Annu Rev Plant Biol. 2007:58:219-47.

19. Assmann SM. Enhancement of the Stomatal Response to Blue Light by Red Light, Reduced Intercellular Concentrations of $\mathrm{CO}(2)$, and Low Vapor Pressure Differences. Plant Physiol. 1988;87(1):226-31.

20. Bergmann DC, Sack FD. Stomatal development. Annu Rev Plant Biol. 2007;58:163-81 
21. MacArthur BD, Ma'ayan A, Lemischka IR. Systems biology of stem cell fate and cellular reprogramming. Nat Rev Mol Cell Biol. 2009;10(10):672-81.

22. Steinway $\mathrm{SN}$, et al. Network modeling of TGFbeta signaling in hepatocellular carcinoma epithelial-to-mesenchymal transition reveals joint sonic hedgehog and Wnt pathway activation. Cancer Res. 2014;74(21):5963-77.

23. Saadatpour A, Albert R, Reluga TC. A Reduction Method for Boolean Network Models Proven to Conserve Attractors. SIAM J Appl Dyn Syst. 2013; 12(4):1997-2011.

24. Ansotegui C, Manya F. Mapping problems with finite-domain variables to problems with Boolean variables. Theory Appl of Satisfiability Test. 2005;3542:1-15

25. Van Ham P. How to deal with more than two levels. In: Thomas R, editor. Kinetic logic : a Boolean approach to the analysis of complex regulatory systems : proceedings of the EMBO course "Formal analysis of genetic regulation," held in Brussels, September 6-16, 1977. Berlin; New York: Springer; 1979. p. 326-44.

26. Didier G, Remy E, Chaouiya C. Mapping multivalued onto Boolean dynamics. J Theor Biol. 2011;270(1):177-84.

27. Karlsson PE. Blue light regulation of stomata in wheat seedlings. I. Influence of red background illumination and initial conductance level. Physiol Plant. 1986;66:5.

28. Zanudo JG, Albert R. An effective network reduction approach to find the dynamical repertoire of discrete dynamic networks. Chaos. 2013; 23(2):025111.

29. Chaouiya C, et al. SBML qualitative models: a model representation format and infrastructure to foster interactions between qualitative modelling formalisms and tools. BMC Syst Biol. 2013;7:135.

30. Veliz-Cuba A, et al. Steady state analysis of Boolean molecular network models via model reduction and computational algebra. BMC Bioinformatics. 2014;15:221.

31. Remy E, Ruet P, Thieffry D. Graphic requirements for multistability and attractive cycles in a Boolean dynamical framework. Adv Appl Math. 2008; 41(3):335-50.

32. Remy E, Ruet P. On differentiation and homeostatic behaviours of Boolean dynamical systems. Lect Notes Bioinformatics. 2007;4780:92-101.

33. Richard A, Comet J-P. Necessary conditions for multistationarity in discrete dynamical systems. Discret Appl Math. 2007;155(18):2403-13.

34. Richard A. Negative circuits and sustained oscillations in asynchronous automata networks. Adv Appl Math. 2010:44(4):378-92.

35. Naldi A, et al. Dynamically consistent reduction of logical regulatory graphs. Theor Comput Sci. 2011;412(21):2207-18.

36. Liu YY, Slotine JJ, Barabasi AL. Controllability of complex networks. Nature. 2011;473(7346):167-73.

37. Lin CT. Structural controllability. IEEE Trans Autom Control. 1974;AC19(3):201-8.

38. Mochizuki A, et al. Dynamics and control at feedback vertex sets. II: A faithful monitor to determine the diversity of molecular activities in regulatory networks. J Theor Biol. 2013;335:130-46.

39. Zanudo JG, Albert R. Cell fate reprogramming by control of intracellular network dynamics. PLoS Comput Biol. 2015;11(4):e1004193.

40. Schwartz A, et al. Anion-Channel Blockers Inhibit S-Type Anion Channels and Abscisic Acid Responses in Guard Cells. Plant Physiol. 1995;109(2):651-8.

41. Kim TH, et al. Guard cell signal transduction network: advances in understanding abscisic acid, $\mathrm{CO} 2$, and $\mathrm{Ca} 2+$ signaling. Annu Rev Plant Biol. 2010;61:561-91.

42. Albert $\mathrm{R}$, Othmer HG. The topology of the regulatory interactions predicts the expression pattern of the segment polarity genes in Drosophila melanogaster. J Theor Biol. 2003;223(1):1-18.

\section{Submit your next manuscript to BioMed Central and we will help you at every step:}

- We accept pre-submission inquiries

- Our selector tool helps you to find the most relevant journal

- We provide round the clock customer support

- Convenient online submission

- Thorough peer review

- Inclusion in PubMed and all major indexing services

- Maximum visibility for your research

Submit your manuscript at www.biomedcentral.com/submit
Biomed Central 